https://helda.helsinki.fi

\title{
Interaction points in plant stress signaling pathways
}

\section{Overmyer, Kirk Loren}

2018-02

Overmyer , K L , Vuorinen , K E \& Brosche , M J 2018 , ' Interaction points in plant stress

pÿsignaling pathways ' , Physiologia Plantarum , vol. 162 , no. 2 , pp. 191204 . https://doi.org/10.1111/ppl.12639

http://hdl.handle.net/10138/324092

https://doi.org/10.1111/ppl.12639

acceptedVersion

Downloaded from Helda, University of Helsinki institutional repository.

This is an electronic reprint of the original article.

This reprint may differ from the original in pagination and typographic detail.

Please cite the original version. 


\title{
Interaction points in plant stress signaling pathways
}

\author{
Kirk Overmyera, Katariina Vuorinen ${ }^{\text {a }}$, Mikael Broschéa,b,*
}

a'Division of Plant Biology, Department of Biosciences, Viikki Plant Science Centre, University of Helsinki, P.O. Box 65 (Viikinkaari 1), FI-00014 Helsinki, Finland

${ }^{b}$ Institute of Technology, University of Tartu, Nooruse 1, 50411 Tartu, Estonia

*Corresponding author, e-mail: mikael.brosche@helsinki.fi

Plants live in a world where they are challenged by abiotic and biotic stresses. In response to unfavorable conditions or an acute challenge like a pathogen attack, plants employ various signaling pathways that regulate expression of defense genes and other mechanisms to provide resistance or stress adaptation. Identification of the regulatory steps in defense signaling has seen much progress in recent years. Many of the identified signaling pathways show interactions with each other, exemplified by the modulation of the jasmonic acid response by salicylic acid. Accordingly, defense regulation is more appropriately thought of as a web of interactions, rather than linear pathways. Here we describe various regulatory components and how they interact to provide an appropriate defense response. One of the common assays to monitor the output of defense signaling, as well as interaction between signaling pathways, is the measurement of altered gene expression. We illustrate that, while this is a suitable assays to monitor defense regulation, it can also inadvertently provide overstated conclusions about interaction among signaling pathways.

Abbreviations: $-{ }^{1} \mathrm{O}_{2}$, singlet oxygen; ABA, abscisic acid; aba3, abscisic acid deficient 3 ; abil, abscisic acid insensitive 1; acd, accelerated cell death; agbl, GTP binding protein betal; ARGOS, AUXINREGULATED GENE INVOLVED IN ORGAN SIZE; BAK1, BRI1-ASSOCIATED RECEPTOR KINASE1; BIK1, BOTRYTIS-INDUCED KINASE1; BOI1, BOS1 INTERACTOR1; BOS1, BOTRYTIS SENSITIVE1; CERK1, CHITIN ELICITOR RECEPTOR KINASE1; COI1, CORONATINE INSENSITIVE1; DAMP, damage associated molecular pattern; EDS1, ENHANCED DISEASE SUSCEPTIBILITY1; EFR, ELONGATION FACTOR-TU RECEPTOR; EIN2, ETHYLENE INSENSITIVE2; ERF6, ETHYLENE RESPONSIVE ELEMENT BINDING FACTOR6; ET, ethylene; ETI, effector triggered immunity; FLS2, FLAGELLIN-SENSITIVE2; GA, gibberellic acid; $\mathrm{H}_{2} \mathrm{O}_{2}$, hydrogen peroxide; HO•, hydroxyl radical; HR, hypersensitive response; HSFA4, HEAT SHOCK FACTOR A4A; JA, jasmonic acid; JAZ, JASMONATE-ZIM-DOMAIN; lsd, lesions simulating disease resistance; MAP, 
MITOGEN ACTIVATED PROTEIN; MeJA, methylJA; NADPH, nicotinamide adenine dinucleotide phosphate; NO, nitric oxide; NPR1, NONEXPRESSER OF PR GENES1; $\mathrm{O}_{2}{ }^{\circ}$, superoxide; $\mathrm{O}_{3}$, ozone; OPDA, oxo-phytodienoic acid; PAD4, PHYTOALEXIN DEFICIENT4; PAMP, pathogen associated molecular pattern; PDF1.2, PLANT DEFENSIN1.2; PR-1, PATHOGENESIS-RELATED GENE1; PTI, PAMP-triggred immunity; qPCR, real time quantitative reverse transcription PCR; RAP2.6, RELATED TO AP2 6; RBOH, RESPIRATORY BURST OXIDASE HOMOLOGUE; RLK, receptor like kinase; ROS, reactive oxygen species; SA, salicylic acid; SAG101, SENESCENCE-ASSOCIATED GENE101; SID2, SALICYLIC ACID INDUCTION DEFICIENT2; TF, transcription factor; XLG2, EXTRA-LARGE GTPBINDING PROTEIN2. 


\section{Introduction}

Signaling pathways interact in controlling and integrating all aspects of plant biology including plant development and stress signaling. In their natural environment plants experience a multitude of different growth conditions including fluctuating temperature, light, water and nutrient availability, air pollutants, and attack by pathogens and insects. In response to a sudden environmental change, plants rely on multiple signaling pathways to mount efficient defense responses with the ultimate aim to adapt and survive under suboptimal conditions. Different proteins and small molecules participate in defense signaling including MITOGEN ACTIVATED PROTEIN (MAP) kinases, transcription factors (TFs), reactive oxygen species (ROS), nitric oxide (NO) and $\mathrm{Ca}^{2+}$, and the hormones abscisic acid (ABA), ethylene, jasmonic acid (JA) and salicylic acid (SA; (Mignolet-Spruyt et al., 2016). Each of these components regulate specific branches in defense signaling, thus for optimized defense responses to occur it is likely that several signaling pathways are activated and interact to execute appropriate responses. Furthermore, the defense responses to one stress (e.g. pathogen infection) may be inappropriate for another (e.g. insect attack). Perhaps the best characterized output from defense signaling is altered gene expression, full genome transcriptome data is available for a vast number of biotic and abiotic experiments and can be studied in databases like Genevestigator (https://genevestigator.com/gv/). Furthermore, a small number of marker genes including PR-1 (PATHOGENESIS-RELATED GENE1), a pathogen and SA induced gene, have gained near universal status as a marker to test for activation of SA signaling (van Loon et al., 2006), nowadays often done with real time quantitative reverse transcription PCR (qPCR). Typically, a full genome gene expression experiment will identify hundreds to thousands of differentially expressed genes. However, we know comparably less about the signal transductions pathways and TFs that regulate these changes in gene expression.

While progress has been made in the identification of regulatory components for many different stress signal pathways, there is still a lack of information especially for the first perception of a stress and how signal pathways are initially activated. Here, the best documented defense signaling response is probably the response to pathogen attack, where both initial perception of the pathogen by receptor like kinases (RLKs) as well as activation of ROS, hormonal and kinase signaling cascades has been studied in great detail (Asai et al., 2002; Kim et al., 2014b; Kimura et al., 2017; Sun et al., 2013). The principle of multi-layered defense signaling is also illustrated by the response to pathogens: initial defense responses depend on pathogen associated molecular patterns (PAMP)-triggered immunity (PTI), where conserved structures, such as the bacterial flagellin peptide, flg22, are recognized by RLKs. Similar to PAMPs, molecular signatures of the damaged plant structures, so called damage associated molecular patters (DAMPs), can also be recognized by RLKs and trigger immunity. Some pathogens have developed effectors to block PTI responses, and in turn plants can recognize these effectors leading to effector triggered immunity (ETI) and mounting of efficient defenses (Dong et al., 2015; Li et al., 2016). 
Interaction points must also exist between different abiotic stresses and between abiotic-biotic stresses, since for example the stress hormone ABA, a critical component in defenses related to cold, drought, and osmotic stress (Zhu, 2016), is also a regulator in the defense against the necrotrophic fungi Botrytis cinerea (Liu et al., 2015). Indeed, several plant hormone signaling pathways feature prominently in stress interactions. Our knowledge of plant stress signaling pathways is to a large extent derived from the model plant Arabidopsis thaliana, and especially mutant screens have yielded key insights. This includes the identification of the JA receptor COI1 (CORONATINE INSENSITIVE1), the essential ethylene signaling component EIN2 (ETHYLENE INSENSITIVE2) and the SA co-transcriptional regulator NPR1 (NONEXPRESSER OF PR GENES1) (Robert-Seilaniantz et al., 2011). Here we review interaction points in defense signaling and components that integrate and regulate different signaling pathways, with a focus on what we have learned from Arabidopsis.

\section{ROS - a common signal molecule in defense signaling}

ROS are highly reactive molecules that include hydrogen peroxide $\left(\mathrm{H}_{2} \mathrm{O}_{2}\right)$, superoxide $\left(\mathrm{O}_{2}{ }^{\circ}\right)$, singlet oxygen $\left({ }^{1} \mathrm{O}_{2}\right)$, and hydroxyl radical $(\mathrm{HO} \bullet)$. Inside the cell ROS are produced during photosynthesis in chloroplasts and in metabolic reactions in the mitochondria and peroxisomes. In these basic metabolic reactions ROS are quickly quenched in order to protect compounds including lipids, protein and DNA from harmful oxidation. In the apoplast ROS are mainly produced by NADPH oxidases (in plants known as RESPIRATORY BURST OXIDASE HOMOLOGUEs, RBOHs) and cell wall peroxidases. Although the traditional view of ROS is as harmful agents, they are now acknowledged as critical signaling intermediates in regulation of development and stress (Vaahtera et al., 2014). ROS signaling rarely functions alone; rather acting together with other signaling pathways, especially those mediated by plant hormones SA, JA, ABA and ethylene (MignoletSpruyt et al., 2016). Many of the regulatory proteins in ROS signaling are the same as those identified from e.g. pathogen-induced signaling pathways and include MAP (MITOGEN ACTIVATED PROTEIN) kinases MPK3 and MPK6 (Ahlfors et al., 2004), and TFs including ERF6 (ETHYLENE RESPONSIVE ELEMENT BINDING FACTOR6) (Meng et al., 2013; Xu et al., 2015a). Importantly, ROS are regulators in both intracellular signaling and long distance cell to cell signaling (Fig. 1); (Miller et al., 2009).

The large overlap between ROS- and pathogen-induced signaling can at least partially be explained by the ROS burst induced by the plant upon recognition of an ongoing pathogen attack (Vaahtera et al., 2014). One convenient tool to study the regulatory role of this ROS burst is to treat plants with the air pollutant ozone $\left(\mathrm{O}_{3}\right)$ - which breaks down to $\mathrm{O}_{2}{ }^{-}$and $\mathrm{H}_{2} \mathrm{O}_{2}$ in the apoplast, and thus mimics a pathogen induced $\mathrm{RBOH}$ and/or peroxidase mediated ROS burst (Vaahtera et al., 2014). $\mathrm{O}_{3}$ treatments allow the study of apoplastic ROS and downstream signaling without the confounding factors of pathogen derived effectors, which would inevitably be part of an experimental design using a pathogen to initiate ROS signaling pathways.

Early events after $\mathrm{O}_{3}$ treatment include activation of MPK signaling and altered gene expression (Ahlfors et al., 2004; Xu et al., 2015a). Furthermore, $\mathrm{O}_{3}$ initiates cell death signaling, which in sensitive genotypes 
ultimately leads to large scale tissue collapse (Brosche et al., 2010; Xu et al., 2015a). The extent of cell death is experimentally easy to measure, hence through the use of Arabidopsis mutants defective in one or more signaling components it becomes possible to determine how different signaling components interact with ROS signaling. SA and ROS can form a positive interaction loop that promotes cell death, but at the same time SA is required to initiate defense signaling (Xu et al., 2015b). Hence, SA can be thought to act at the balance between too little and too strong activation of defenses. In $\mathrm{O}_{3}$-activated cell death, ethylene promotes cell death and JA protects against it, both showing mutual inhibitory effects (Fig. 1); (Tuominen et al., 2004; $\mathrm{Xu}$ et al., 2015a). The inhibition of JA signaling by ethylene is also observed during PTI signaling (Kim et al., 2014). Further highlighting the complexity in signal interaction, ethylene and JA also need to act together to regulate expression of some marker genes (Penninckx et al., 1998). In addition, the extent of the interaction between ROS and hormones also depends on which experimental parameter is measured. In contrast to the clear interplay between ROS, JA and ethylene in the regulation of cell death, if instead gene expression is measured, the majority of transcriptome changes elicited by $\mathrm{O}_{3}$ are hormone independent $(\mathrm{Xu}$ et al., 2015a). This indicates that ROS also directly activate signal transduction pathways through e.g. oxidative posttranscriptional modifications (mostly on cysteine groups) and activation of kinases (Kimura et al., 2017).

\section{Interactions between SA - JA and beyond}

In plant defense signaling the interactions between the hormones SA and JA are probably the most well studied (Caarls et al., 2015). Typically, SA-mediated defenses are effective against pathogens with a biotrophic lifestyle, which require live host cells, whereas JA mediated defenses protect against insects or pathogens with a necrotrophic lifestyle, which kill host cells and derive nutrients from dead tissues.

Multiple lines of evidence demonstrate that activation of SA signaling leads to the inhibition of JA signaling, both at the level of gene expression and ultimately plant immunity (Caarls et al., 2015; Spoel et al., 2007). A commonly used assay relies on treatments with MeJA (methylJA) to activate increased expression of the JAresponsive marker gene PDF1.2. Simultaneous application of MeJA with SA blocks the induction of PDF1.2 expression, and based on mutant analysis several proteins have been shown to regulate this interaction either directly, e.g. ORA59, or indirectly e.g. WRKY70, MYB44, TGA2, TGA5, TGA6 and NPR1 (Caarls et al., 2015). Most of the regulators identified to date are either TFs or co-transcriptional regulators. Furthermore, the GCC-box promoter element bound by ORA59 is important for SA inhibition of JA signaling. A change in the redox balance of the cell is required as a signal up-stream from TFs for the SA-JA inhibition, since treatments with a glutathione biosynthesis inhibitor, as well as overexpression of a glutaredoxin GRX480 can interfere with PDF1.2 regulation (Caarls et al., 2015). Furthermore, the co-transcriptional regulator NPR1, essential for proper SA responses, is regulated by the cellular redox balance and numerous post-translational modifications including S-nitrosylation, sumoylation, and phosphorylation (Withers and Dong, 2016). 
Intriguingly, another stress hormone, ABA, also directly targets the accumulation of NPR1, hence representing an inhibitory action of ABA on SA signaling (Ding et al., 2016a).

Beyond the inhibitory action of SA on JA signaling, recently a positive interaction between these hormones was described. Activation of ETI signaling after infection with Pseudomonas syringae pv maculicola ES4326/avrRpt2 leads to increased production of both SA and JA (Liu et al., 2016). The increase in expression of JA-responsive genes required SA biosynthesis and the SA receptors NPR3 and NPR4 could directly regulate the stability of the JA repressor JAZ (JASMONATE-ZIM-DOMAIN) proteins (Liu et al., 2016). Thus, SA can directly modify JA signaling by bypassing the classical JA - COI1 signaling pathway and instead act on the JAZ repressors to initiate JA signaling.

To fully explore the interaction between SA and JA, as well as their interaction with ethylene and a defense regulator PAD4 (PHYTOALEXIN DEFICIENT4) a quadruple mutant (dde2 ein2 pad4 sid2) deficient in SA (sid2), JA (dde2), ethylene (ein2) and pad4 was analyzed in response to PTI, ETI and a necrotrophic fungi (Alternaria brassicicola; (Hillmer et al., 2017; Kim et al., 2014; Tsuda et al., 2009). In parallel all possible single, double and triple mutants were also analyzed. Strikingly, while all three hormones contribute to robust plant-immunity, the interaction is synergistic in PTI, which possibly facilitates signal amplification, whereas in ETI the interaction between hormones is compensatory, i.e. when one hormone signal pathway is lost - the remaining active signaling pathways can still activate defenses (Tsuda et al., 2009). Interactions between the hormones were also found, ethylene acts as an inhibitor of JA (and PAD4) signaling (Kim et al., 2014), which is similar to the inhibitory effect of ethylene on JA signaling seen in $\mathrm{O}_{3}$-regulated cell death (Tuominen et al., 2004; Xu et al., 2015a). Furthermore, JA signaling can also activate SA signaling (Kim et al., 2014), highlighting that both co-operation and antagonism between signaling pathways are possible. High resolution time series analysis of flg22 transcriptome changes in the same set of single and higher order mutants was used to estimate the degree of co-operation and interactions among the stress hormones and PAD4 (Hillmer et al., 2017). Strikingly, flg22 activation of SA signaling is indirect, and requires prior activation of JA, ethylene and PAD4 signaling (Hillmer et al., 2017). The specific interaction seen in response to any given treatment is likely context dependent. Presumably, the interaction between different signaling pathways (like the SA-JA interaction) is dependent on the specific stress or growth conditions and provides plants with a robust defense-signaling network, capable of adapting to the environment.

Fig. 1 reveals both the complexity of hormonal interactions and an inherent limitation of interaction studies. It seems with a broad enough literature search, it is likely to find at least one example of a given interaction going the opposite way to what was initially expected. One challenge in this research area, is the deconvolution of this apparent complexity, which is likely caused by several factors including differences in read outs and methods used, different experimental designs, and importantly, studies considering different time scales.

\section{The role of kinases}


One of the earliest events after perception of abiotic and biotic stress is increased activity of mitogenactivated protein (MAP) kinase cascades consisting of a MAPKKK (MAPK kinase kinase), a MAPKK (MAPK kinase) and a MAP kinase (Fig. 1). Especially MPK3, MPK4 and MPK6 are activated in response to many stresses (Meng and Zhang, 2013). Furthermore, MPK kinases are also regulated by subcellular relocalization, for example MPK3 and MPK6 are rapidly activated by $\mathrm{O}_{3}$ treatment and translocated from the cytosol to the nucleus (Ahlfors et al., 2004). The increased MPK activity seen in response to most stresses indicate that they perform a general function required for plant defenses. Activated MAP kinases phosphorylate and regulate the activity of target proteins. Several MPK target-proteins are TFs, including MPK4-WRKY33, MPK3/MPK6-WRKY33, MPK3/MPK6-ERF6, MPK3/MPK6-HSFA4 (HEAT SHOCK FACTOR A4A) and MPK6-ZAT6, implying that one important role for MPK signaling is regulation of transcriptional responses in response to stress (Li et al., 2016; Liu et al., 2013; Meng et al., 2013; PerezSalamo et al., 2014). This is supported by the identification of numerous mis-regulated transcripts in $m p k 3$, $m p k 4$ and $m p k 6$ mutants (Frey et al., 2014). Furthermore, signaling through MPK3 can confer both SAdependent and -independent regulation of SA-responsive genes (Genot et al., 2017; Tsuda et al., 2013). However, since MPKs are activated in response to most stresses, this also raises the question of how stressspecific responses are achieved? Most likely several signaling pathways are activated in parallel in response to stress, and the interactions among the signaling pathways leads to a stimulus specific response.

Initial stress perception is often mediated by receptor-like kinases (RLKs). In Arabidopsis there are $>600$ RLKs and they are involved in perception of both abiotic and biotic stresses (Bourdais et al., 2015; Kimura et al., 2017). The best characterized defense-related RLK is probably FLAGELLIN-SENSITIVE 2 (FLS2) that detects bacterial flagellin as part of pathogen perception that leads to PTI signaling. A co-receptor BRI1ASSOCIATED RECEPTOR KINASE1 (BAK1) is required for FLS2, as well as several other RLKs, to initiate down-stream signaling (Sun et al., 2013). Perception of flg22 by FLS2 leads to activation of MPK3, MPK4 and MPK6, raising the possibility that an RLK (FLS2), directly or indirectly activates MPK cascades (Asai et al., 2002). Recently, FLS2 was shown to interact with and phosphorylate the MAPKKK MKKK7 (Mithoe et al., 2016). However, MKKK7 was shown to be a negative regulator of MPK activity and defense signaling, hence direct mechanistic insight into how MPK cascades are activated and the possible role of RLKs is missing.

Activation of FLS2 also leads to a ROS burst through BIK1 (BOTRYTIS-INDUCED KINASE1) and activation of RBOHD ( $\mathrm{Li}$ et al., 2014). Activation of RBOHs is one common signal interaction point since multiple other kinases, including several activated by $\mathrm{Ca}^{2+}$ also regulate RBOHD and RBOHF activity (Wrzaczek et al., 2013). Furthermore, other proteins also influence ROS production including EXTRALARGE GTP-BINDING PROTEIN2 (XLG2) that interacts with FLS2 and BIK1 to regulate ROS production (Liang et al., 2016). Overall kinases from different gene families appear to be crucial both in the initial perception of stress, integrating information from several sources, and transmitting the signal onto TFs to regulate gene expression (Fig. 1). 


\section{EDS1 and PAD4 - coordinators of defense signaling}

ENHANCED DISEASE SUSCEPTIBILITY1 (EDS1) and PAD4 are two sequence related proteins that regulate pathogen responses, cell death, abiotic-stress tolerance, and SA-dependent and -independent defense signaling (Cui et al., 2017; Wituszynska et al., 2013). Initial characterization placed EDS1 as a crucial regulator of signaling in ETI (Wiermer et al., 2005). This also includes regulation of SA accumulation after pathogen infection. However, the function of EDS1 goes far beyond the regulation of ETI signaling. For example the eds 1 mutation alone or in combination with the SA biosynthesis mutant sid 2 reduced cell death in a variety of signaling contexts (Kaurilind et al., 2015; Straus et al., 2010; Venugopal et al., 2009), placing EDS1 as a prominent regulator of cell death together with SA. EDS1 forms alternate complexes with PAD4 or SENESCENCE-ASSOCIATED GENE101 (SAG101), separately in the cytosol and in the nucleus (Fig. 1). The direct molecular function of EDS1 is thought to be associated with transcriptional regulation in the nucleus and cell-death regulation in the cytosol (Heidrich et al., 2011). SA can also increase the expression of EDS1 and PAD4, leading to an enhanced signal output (Cui et al., 2017). Furthermore, EDS1 and PAD4 can regulate pathogen-induced gene expression both in parallel to, and cooperatively with SA. This ensures robust activation of defense gene expression, i.e. if one of the pathways would be blocked redundancy between the pathways would still ensure that defenses are activated (Cui et al., 2017). In abitotic stress responses EDS1, PAD4 and SAG101 are required for proper responses to freezing stress through regulation of SA, ROS and lipid levels (Chen et al., 2015). Since functional EDS1 is needed for proper defense responses in several different contexts (Kaurilind et al., 2015; Straus et al., 2010; Wang et al., 2013; Venugopal et al., 2009), it represents one important signal interaction point (Fig. 1).

\section{Transcription factors as signal integrators}

As the direct regulators of gene expressions, TFs are often found to integrate signals from different signaling pathways. As an example of this, below, we consider the role of TFs in mediating the sometimes surprising hormone- and stress-interactions between wounding, immunity, and cell death.

The TF WRKY33 regulates expression of ABA biosynthesis genes to influence the outcome of Botrytis infection (Liu et al., 2015). In addition, WRKY33 is a target of MPK signaling (Qiu et al., 2008; Rasmussen et al., 2012). BOS1 (BOTRYTIS SENSITIVE1) encodes a R2R3 type MYB transcription factor (MYB108) and was first characterized for its role in susceptibility to Botrytis infection (Mengiste et al., 2003). In addition to Botrytis sensitivity, loss of BOS1 function results in enhanced symptom formation (cell death) in response to abiotic stress, ROS treatments, and infection with virulent and avirulent bacterial pathogens (Mengiste et al., 2003). Furthermore, BOS1 modulates a wound-induced spreading cell death program involved in sealing wounds (Cui et al., 2013). Wounding the bos 1 mutant resulted in a run-away spreading cell-death phenotype eventually spreading throughout the whole plant, while in wild-type Col-0 plants only a small number of cells died, encompassing only a few rows of cells immediately adjacent to the cells disrupted by the wound. This cell death program is reminiscent of lesions simulating disease resistance (lsd) 
and accelerated cell death (acd) mutants that ectopically activate hypersensitive response (HR)-like cell death and immune signaling pathways (Bruggeman et al., 2015). In particular, bos 1 is similar to the $l s d l$ mutant; both of these mutants have runaway cell-death phenotypes indicating that they encode negative regulators of cell-death propagation. However, in contrast to the $l s d$ and acd mutants, cell death in bos 1 is independent of SA, metacaspases and ROS from RBOHs but requires both ABA biosynthesis and signaling. Indeed, ABA was necessary and sufficient to drive the propagation of wound induced cell death (Cui et al., 2013). BOS1 interacts with and is regulated by the E3 ligase BOS1 INTERACTOR1 (BOI1), the defining member of a small subclade of functionally redundant proteins (Luo et al., 2010). Plants bearing RNA $i$ constructs that target the members of the BOI1 subclade exhibited transcriptional profiles indicative of an enhanced gibberellic acid (GA) response. Taken together, the above results suggest that BOS1 regulates cell death via modulation of the balance between GA and ABA signaling, two hormones known to regulate cell death in several plant tissues in a mutually antagonistic fashion (Weiss and Ori, 2007).

Studies with both WRKY33 and BOS1 implicate a role for ABA in the response to Botrytis infection. As a necrotrophic pathogen, Botrytis has the lifestyle and virulence strategy to use toxins and ROS to kill host cells and derive nutrients from dead host tissues. Immunity against necrotrophs is primarily mediated by JAand ethylene-signaling and involves modulation of both defenses and cell death control. Since BOS1 transcription is JA-dependent, it is reasonable to assume that bosl Botrytis-susceptibility is due to loss of some JA-dependent defense responses. Alternatively, loss of cell death control may also explain the susceptibility of bos 1 . Here ABA plays an important role, since it promotes cell death and double mutants with bos1 and ABA signaling (abil, abscisic acid insensitive 1) or biosynthesis (aba3, abscisic acid deficient3) limit the extent of cell death (Cui et al., 2013). This suggests that the plant wound-response can be hijacked by Botrytis to promote virulence. Indeed, Botrytis essentially creates a wound at the primary infection site by deploying toxins and cell wall degrading enzymes to kill and macerate host tissue. ABAand OPDA-responsive specific marker genes, known to have a role in the wound response, are induced during the Botrytis infection (Sham et al., 2015. Remarkably, Botrytis itself is well known to produce ABA (Sharon et al., 2007; Siewers et al., 2006; Siewers et al., 2004; Taki et al., 2005; Windram et al., 2012) and its biosynthesis is induced upon contact with host plants (Kettner and Dorffling, 1995). Furthermore, several studies support the role of ABA in promoting virulence in plant-Botrytis interactions. ABA overproduction has been associated with enhanced virulence in some strains (Ding et al., 2016b; Gong et al., 2014; Siewers et al., 2004) and treatment with exogenous ABA was shown to promote virulence (Shaul et al., 1996). The WRKY33 transcription factor is required for the downregulation of plant ABA biosynthesis and thus promote immunity against Botrytis (Liu et al., 2015). The ABA-deficient sitiens mutant of tomato exhibits enhanced immunity against Botrytis infection (Asselbergh et al., 2007). Botrytis immunity in sitiens tomato was proposed to involve the known antagonism between ABA and SA, due to de-repression of SA-mediated basal defenses. ABA may also directly target components of SA-signaling (Ding et al., 2016a). Further illustrating the complexity in signal interaction (Fig. 1), ABA also inhibits JA and ethylene signaling, and 
reciprocally ethylene inhibits ABA signaling (Anderson et al., 2004). One complicating issue in the study of the role of $\mathrm{ABA}$ and its interaction with other signaling pathways is its role in formation of the cuticle that covers leaves and forms the primary protective barrier to the environment. For reasons that are not yet entirely understood, plants with permeable cuticles are highly immune against Botrytis infection (Bessire et al., 2007). Both ABA-biosynthesis and -signaling are required for cuticle formation (Cui et al., 2016; Serrano et al., 2014), thus mutants with deficient ABA-responses are all cuticle permeable and Botrytis resistant. Cuticle permeable mutants also exhibit enhanced ROS production (Serrano et al., 2014). It has been suggested that components of the unassembled cuticle may act as DAMPs and activate immunity (Serrano et al., 2014). In support of this, wounding, mechanical stimulation, or treating plants with cutinase, result in ROS production and immunity (Serrano et al., 2014). Interestingly, ROS and the cuticle appear to have mutual roles in the regulation of each other. Overexpression of the Arabidopsis extracellular peroxidase, PEROXIDASE57, resulted in ROS overproduction and impaired cuticle development and immunity against Botrytis (Survila et al., 2016). Furthermore, application of ABA reduced ROS production and reduced cuticle permeability, thus the interaction between these components is important for proper defense regulation.

\section{Information from transcriptional regulatory networks}

As outlined above many of the proteins that integrate signals from different sources ultimately regulate transcription and include co-transcriptional regulators (e.g. NPR1) and MPKs that regulate the location or activity of TFs. In addition, measuring whole genome transcriptional responses with arrays or RNA-seq is a comparatively easy experimental technique, thus a wealth of transcriptome data is available in various databases (Vaahtera et al., 2014). On their own, these experiments are already useful to find genes differentially expressed in response to a single treatment, however, the full power of these datasets is revealed when they are combined to identify stimulus specific responses or to predict regulatory networks (Vaahtera et al., 2014; Willems et al., 2016). For instance, ROS were identified as signaling molecules in response to many abiotic and biotic stresses; but the combined analysis of 79 ROS and redox related array experiments identified RBOHF as one key factor in ROS signaling (Willems et al., 2016).

Due to the complete gene coverage of transcriptome datasets, they are also suitable to combine with other "omics" data, including protein-protein interaction, TF-DNA interaction, and chromatin-modification data sets to build regulatory networks. One such network, constructed for ETI and PTI (Dong et al., 2015), revealed multiple TFs important for immune responses and highlighted the crucial role of kinases in defense signaling.

\section{Sequential or simultaneous stress combinations}

Most large scale gene expression experiments in Arabidopsis using either arrays or RNA-seq have focused on a single treatment in controlled chambers or growth rooms. This situation is very different from the environment plants would experience in nature, where growth conditions are more variable and multiple stresses may occur simultaneously. Especially events of combined heat and drought stress or heat and high 
light stress are severely damaging to plants (Zandalinas et al., 2017). In response to multiple stresses at least two scenarios are possible, the response to combined treatment could be more similar to one of the individual stresses or that the combined treatment will lead to a new response that could not have been predicted from the response to a single stress treatment.

In Arabidopsis several studies have estimated the effect of combined drought and heat stress or other abiotic stress combinations on regulation of gene expression (Rasmussen et al., 2013; Zandalinas et al., 2017). Several of these stress combinations lead to a transcriptional response that differs substantially from that elicited by each single treatment (Rasmussen et al., 2013; Zandalinas et al., 2017). This demonstrates that plants have the capacity to integrate multiple signaling pathways to activate an appropriate defense program. One possible regulator in multiple stress interactions is $\mathrm{ABA}$, whose production typically is increased in diverse abiotic-stresses, and whose signaling can modify SA-dependent defenses (Ding et al., 2016a; Rejeb et al., 2014).

A complementary experiment to combined stress treatments, is to perform sequential treatments. This has been done with drought, Botrytis infection, and herbivory (Pieris rapae). Transcriptional responses were measured first after the single treatments and again measured after application of the second stress (Coolen et al., 2016). In all cases, the transcriptional response of the plant follows the last stress encountered, although at early time points there is still some "memory" of the first stress (Coolen et al., 2016). Overall, experiments with combined- and sequential-stresses emphasize that plants are able to integrate and prioritize between different signaling pathways, suggesting further studies with combined stresses may reveal new regulators potentially useful in breeding stress tolerant plants.

\section{Lessons from the use of marker genes}

Signal interactions can be studied by several experimental methods, including whole plant phenotypes e.g. quantification of pathogen growth or the extent of cell death after $\mathrm{O}_{3}$-treatments (Cui et al., 2013; Xu et al., 2015a). However, due to the technical ease of extracting RNA, one of the most popular methods to study the output of signaling pathways is to measure gene expression, either at a global scale with arrays or RNA-seq or more targeted with real-time quantitative reverse-transcription PCR (qPCR) (Vaahtera et al., 2014). A key issue, if using qPCR, is the selection of appropriate marker-genes. A good marker gene should be as specific as possible, that is, show altered expression in response only to the treatment of interest. Unfortunately very few stress regulated genes are uniquely regulated by only one stress (Vaahtera et al., 2014). Selection of suitable marker genes should take advantage of the extensive microarray and RNA-seq data available in the public domain (accessible for example in a user friendly format in Genevestigator; https://genevestigator.com/gv/). By comparison of different biotic and abiotic stresses with hormone and other treatments, it becomes possible to judge to which signal pathway the intended marker genes belongs to. Since Genevestigator also displays data from many different variants of the same experiment (e.g. currently there are multiple different ABA experiments in Genevestigator for Arabidopsis), it becomes possible to 
judge how robust the response of a marker gene is across different experimental set-ups. In many cases it might not be possible to find a single marker gene that shows the desired expression profile, instead it may be possible to use several marker genes that together will show which signal pathways are activated (Vaahtera et al., 2014). After carefully selecting marker genes, a gene expression experiment also requires careful considerations in the interpretation of the results (Vaahtera et al., 2014). This problem is illustrated in Fig. 2 using an example qPCR experiment with wildtype and mutant, in control and treatment. In Fig. 2A the qPCR data is presented for all samples scaled to the wildtype control, while in Fig. $2 \mathrm{~B}$ the same data is recalculated to show the fold-change caused by the treatment in the wildtype and in the mutant.

As presented in Fig. 2B, the data suggests the mutation has no effect on gene expression, while as seen in Fig. 2A, the overall level of gene expression is five-fold lower in the mutant. Hence, the better way to present such data is to display all samples (as in Fig. 2A) or to display the data both ways. A published example of this, was the analysis of transgenic Arabidopsis expressing a gain-of-function variant of the ERF6 TF after $\mathrm{O}_{3}$ treatment (Xu et al., 2015a). In gene expression data displayed as fold inductions, this mutant appeared to be severely impaired in $\mathrm{O}_{3}$-responses, but the data for both control and $\mathrm{O}_{3}$-treatment revealed that this apparent deficiency was due to very high defense gene expression already in control conditions.

To further illustrate this principle and it's effect on interpretation of signal interactions, we performed a qPCR experiment after one hour of $\mathrm{O}_{3}$ to exemplify various interactions between $\mathrm{ROS}$ and hormone signaling. We used the following mutants: ein2 and etr1-1 (defective in ethylene signaling), coil-16 (defective in JA signaling), $N a h G$ and $n p r l$ (defective in SA-accumulation or -signaling), tga 2 tga5 tga6 (defective in three TGA TFs involved in SA- and JA-signaling) and the coil ein2 sid2 eds 1 quadruple mutant defective in multiple pathways, (Xu et al., 2015a). As marker genes we used WRKY38 and PR-1 (early and late SA marker genes), RAP2.6 (a JA marker gene; (Krishnaswamy et al., 2011), ARGOS (an ethylene marker gene; (Xu et al., 2015a), PDF1.2 (a classical JA and ethylene marker gene) and ZAT12 (a general ROS marker gene; (Davletova et al., 2005).

Expression of WRKY38, ARGOS, and RAP2.6 was increased by the $1 \mathrm{hr} \mathrm{O}_{3}$ treatment, directly demonstrating that apoplastic ROS activates SA, ethylene and JA signaling (Fig. 3). This activation was blocked by mutations that disrupt hormone signaling or accumulation: ARGOS expression was reduced in ein2, WRKY38 expression in NahG and RAP2.6 expression in coil-16 (Fig. 3). Expression of WRKY38 is also impaired in $n p r 1$, but this was more clearly seen at later time points of $\mathrm{O}_{3}$ treatment (Brosche et al., 2014). Flg22 induced expression of $A R G O S$ is also abolished in ein2 (Hillmer et al., 2017), indicating the similarities between $\mathrm{O}_{3}$ and flg22 signaling (Vaahtera et al., 2014). The classically used marker genes $P R-1$ and PDF1.2 did not have significantly altered expression after $1 \mathrm{hr}_{3}$ treatment, suggesting that they are not suitable marker genes for the study of early ROS signaling. 
Presenting qPCR data only as fold inductions (Fig. 3) obscures one prominent interaction - the inhibition of SA signaling by ethylene. In Fig. 4 (displaying relative expression values scaled to the Col-0 control), the expression of the SA markers $P R-1$ and WRKY38 was substantially higher in the ein2 mutant. Since SA marker gene expression was elevated under both control and $\mathrm{O}_{3}$, the fold induction in these mutants appeared similar to the wildtype (Fig. 3), hence choices in data presentation may lead to the masking of relevant information. Increased SA-responsive gene expression in ethylene-deficient mutants was previously observed in the regulation of cell death (Plett et al., 2009). Additionally, Fig. 4 also revealed that the ein2 mutant appears to be more strongly impaired in ethylene signaling than the etrl mutant (as also seen in the $A R G O S$ fold induction in Fig. 3). Also, the inhibition of SA responsive genes by ethylene was no longer observed when both ethylene and SA signaling was impaired in the coil ein2 sid2 eds1 mutant. Further complicating the issue, SA may also be required for stress induced activation of ethylene signaling (Fig. 1); (Rao et al., 2002).

The general ROS marker ZAT12 was very highly induced independent of the mutant background, including the quadruple coil ein 2 sid2 eds1. The hormone independent regulation of ZAT12 expression is not a special case, in RNA-seq analysis approximately $70 \%$ of $\mathrm{O}_{3}$-regulated genes were found to have hormoneindependent regulation (Xu et al., 2015a). Similarly, investigation of flg22 responsive genes revealed that one third of them (2659 genes) had hormone-independent regulation (Hillmer et al., 2017). Hence, we face a challenge, but also an opportunity, in finding and understanding the currently unknown signaling pathways that regulate many ROS- and defense-responsive genes.

\section{Conclusions and future directions}

In response to a given stress multiple signaling pathways are activated and the sum of interactions between these lead to a final output that is specifically tailored to counter that stress. Depending on the assay used and genetic background, inhibitory and synergistic interactions can be found between most signaling pathways, especially when considering analyses over different time scales. A future challenge is to determine the relevance of these interactions. The popular qPCR assay, while easy to perform, should be treated cautiously - depending on which marker gene and the genetic background are used and how the data are displayed, this method can be used both to gain insight into signal interaction points, but may also be inadvertently misrepresented to overstate the importance of specific interactions.

In the future it will also be important to determine where the interaction between different signal pathways takes place: Is it through selective import of a transcriptional or co-transcriptional regulator to the nucleus (e.g. EDS1 or NPR1)? Through regulation of protein levels (like ethylene signaling can regulate the accumulation of the FLS2 receptor, (Mersmann et al., 2010))? Or via differential accumulation of stress hormones (Gupta et al., 2017)? It will also be important to evaluate the purpose of the signal interaction. Is it to allow priority between potentially conflicting defense signal pathways or is the interaction aimed at providing flexibility and redundancy to allow timely execution of defense signaling? 


\section{Acknowledgements}

Our research is supported by the Academy of Finland Center of Excellence in Molecular Biology of Primary Producers 2014-2019 (Decisions no. 271832 and 307335) and the University of Helsinki. K.V. is a member of the University of Helsinki Doctoral Programme in Plant Sciences (DPPS). The Scandinavian Society of Plant Physiology (SPPS) support for the early career principle investigator (ECPI) network is gratefully acknowledged.

\section{Figure legends}

Fig. 1. Examples of signal interaction points. At the plasma membrane initial pathogen perception is mediated by receptors (exemplified by FLS2) that recognize conserved pathogen structures (e.g. flg22) to initiate down-stream signaling. This includes activation of ROS-production through RBOHs via XLG2 and various kinases including BIK1. In addition, $\mathrm{Ca}^{2+}$ and $\mathrm{Ca}^{2+}$-activated kinases also regulate $\mathrm{RBOH}$ activity. In turn, ROS can activate both inter- and intra-cellular signaling pathways. In parallel, MPK signaling is also activated, where the targets include phosphorylation of TFs as well as transport of MPKs into the nucleus. Selective transport of various TFs and co-transcriptional regulators is a prominent regulatory step, exemplified by the redox regulated oligomer to monomer step required for import of NPR1 into the nucleus. For EDS1 to act as a transcriptional regulator it also needs to be nuclear localized (Heidrich et al., 2011). Interaction between stress hormones include both positive and negative interactions, but exactly how positive and negative interactions are mediated at the molecular level remains to be fully explored, but is likely to include the selected nuclear transport of transcriptional regulators or their activation, including NPR1, where SA regulates the monomer status of NPR1 via redox modifications (Withers and Dong, 2016). The interaction among hormones is complex, possibly all possible combinations of positive and negative interactions among SA, JA, ABA and ethylene (ET) can be found in the literature. All signal interaction models come with caveats: (1) Due to the ease of experimentally measuring altered gene expression, a large proportion of proposed signal interactions are based mostly only the use of gene expression data with a few selected marker genes. (2) For simplicity the interaction among hormones is often drawn as direct interactions as in Fig. 1, but the interactions may be direct (e.g. two different signaling pathways targeting the same TF) or indirect (e.g. regulation of the amount of a receptor or signaling protein, exemplified by ethylene signaling that regulates the amount of the FLS2 receptor (Mersmann et al., 2010)). (3) In a static model it is difficult to accurately represent the role of time. In this model, events at the plasma membrane and activation of kinases takes place within minutes, whereas gene expression is often measured within a few hours, and later events e.g. outcomes in pathogen tolerance or extent of cell death is measured many hours to days after a treatment. Hence a given interaction, between two or more components, may be positive at early time points but can change to something else at later time points. 
Fig. 2. The raw data from a qPCR experiment consists of cycle values (typically $\mathrm{Ct}$ or equivalent), which are subsequently re-calculated into relative expression (A) or fold induction (B) taking into account reference (or normalization) genes and primer efficiencies. When working with mutants, presenting only treatment fold induction data may inadvertently give misleading information about the mutant phenotype.

Fig. 3. Gene expression was measured with qPCR after a $1 \mathrm{hr} \mathrm{O}_{3}$-treatment as previously described and fold induction calculated with qBase (Brosche et al., 2014; Xu et al., 2015a). Fold change of selected marker genes in ein2 and etrl (ethylene signaling), coil-16 (JA signaling), NahG and nprl (SA signaling), a triple TGA TF mutant tga256, and the quadruple mutant coil ein2 sid2 eds 1 (abbreviated quad) deficient in multiple signaling pathways. The bars represent average and standard deviation of four biological replicates. Statistical analysis was performed with one-way ANOVA and Dunnets multiple comparison test (GraphPad Prism 6.0 on $\log 2$ transformed data), $*>0.05 ; * *>0.01$ denote significant differences from Col-0 wildtype.

Fig. 4. Gene expression was measured with qPCR after a $1 \mathrm{hr} \mathrm{O}_{3}$-treatment as previously described and relative expression calculated with qBase (Brosche et al., 2014; Xu et al., 2015a). Expression of $P R-1$ and WRKY 38 was scaled to the Col-0 control in ein2 and etrl (ethylene signaling) and the quadruple mutant coil ein 2 sid2 eds 1 (abbreviated quad) deficient in multiple signaling pathways. The bars represent average and standard deviation of four biological replicates. Statistical analysis was performed with one-way ANOVA and Dunnets multiple comparison test (GraphPad Prism 6.0 on $\log 2$ transformed data), ** $>0.01$ denote significant differences from Col-0 control.

\section{References}

Ahlfors R, Macioszek V, Rudd J, Brosche M, Schlichting R, Scheel D and Kangasjärvi J (2004) Stress hormoneindependent activation and nuclear translocation of mitogen-activated protein kinases in Arabidopsis thaliana during ozone exposure. Plant J 40:512-522.

Anderson JP, Badruzsaufari E, Schenk PM, Manners JM, Desmond OJ, Ehlert C, Maclean DJ, Ebert PR and Kazan K (2004) Antagonistic interaction between abscisic acid and jasmonate-ethylene signaling pathways modulates defense gene expression and disease resistance in Arabidopsis. Plant Cell 16:3460-3479.

Asai T, Tena G, Plotnikova J, Willmann MR, Chiu WL, Gomez-Gomez L, Boller T, Ausubel FM and Sheen J (2002) MAP kinase signalling cascade in Arabidopsis innate immunity. Nature 415:977-983.

Asselbergh B, Curvers K, Franca SC, Audenaert K, Vuylsteke M, Van Breusegem F and Höfte M (2007) Resistance to Botrytis cinerea in sitiens, an abscisic acid-deficient tomato mutant, involves timely production of hydrogen peroxide and cell wall modifications in the epidermis. Plant Physiol 144:1863-1877.

Bessire M, Chassot C, Jacquat AC, Humphry M, Borel S, Petetot JMC, Metraux JP and Nawrath C (2007) A permeable cuticle in Arabidopsis leads to a strong resistance to Botrytis cinerea. Embo J 26:21582168.

Bourdais G, Burdiak P, Gauthier A, Nitsch L, Salojärvi J, Rayapuram C, Idanheimo N, Hunter K, Kimura S, Merilo E, Vaattovaara A, Oracz K, Kaufholdt D, Pallon A, Anggoro DT, Glow D, Lowe J, Zhou J, Mohammadi O, Puukko T, Albert A, Lang H, Ernst D, Kollist H, Brosche M, Durner J, Borst JW, Collinge DB, Karpinski S, Lyngkjaer MF, Robatzek S, Wrzaczek M, Kangasjärvi J and Consortium C (2015) Large-Scale Phenomics Identifies Primary and Fine-Tuning Roles for CRKs in Responses Related to Oxidative Stress. Plos Genet 11. e1005373. 
Brosche M, Blomster T, Salojärvi J, Cui FQ, Sipari N, Leppala J, Lamminmaki A, Tomai G, Narayanasamy S, Reddy RA, Keinänen M, Overmyer K and Kangasjärvi J (2014) Transcriptomics and Functional Genomics of ROS-Induced Cell Death Regulation by RADICAL-INDUCED CELL DEATH1. Plos Genet 10. e1004112.

Brosché M, Merilo E, Mayer F, Pechter P, Puzorjova I, Brader G, Kangasjärvi J and Kollist H (2010) Natural variation in ozone sensitivity among Arabidopsis thaliana accessions and its relation to stomatal conductance. Plant Cell Environ 33:914-925.

Bruggeman Q, Raynaud C, Benhamed M and Delarue M (2015) To die or not to die? Lessons from lesion mimic mutants. Front Plant Sci 6. 24.

Caarls L, Pieterse CMJ and Van Wees SCM (2015) How salicylic acid takes transcriptional control over jasmonic acid signaling. Front Plant Sci 6. 170.

Chen QF, Xu L, Tan WJ, Chen L, Qi H, Xie LJ, Chen MX, Liu BY, Yu L, Yao N, Zhang JH, Shu WS and Xiao S (2015) Disruption of the Arabidopsis Defense Regulator Genes SAG101, EDS1, and PAD4 Confers Enhanced Freezing Tolerance. Molecular Plant 8:1536-1549.

Coolen S, Proietti S, Hickman R, Davila Olivas NH, Huang PP, Van Verk MC, Van Pelt JA, Wittenberg AHJ, De Vos M, Prins M, Van Loon JJA, Aarts MGM, Dicke M, Pieterse CMJ and Van Wees SCM (2016) Transcriptome dynamics of Arabidopsis during sequential biotic and abiotic stresses. Plant Journal 86:249-267.

Cui FQ, Brosche M, Lehtonen MT, Amiryousefi A, Xu EJ, Punkkinen M, Valkonen JPT, Fujii $\mathrm{H}$ and Overmyer K (2016) Dissecting Abscisic Acid Signaling Pathways Involved in Cuticle Formation. Molecular Plant 9:926-938.

Cui FQ, Brosche M, Sipari N, Tang SJ and Overmyer K (2013) Regulation of ABA dependent wound induced spreading cell death by MYB108. New Phytol 200:634-640.

Cui HT, Gobbato E, Kracher B, Qiu JD, Bautor J and Parker JE (2017) A core function of EDS1 with PAD4 is to protect the salicylic acid defense sector in Arabidopsis immunity. New Phytol 213:1802-1817.

Davletova S, Schlauch K, Coutu J and Mittler R (2005) The zinc-finger protein Zat12 plays a central role in reactive oxygen and abiotic stress signaling in Arabidopsis. Plant Physiol 139:847-856.

Ding Y, Dommel M and Mou Z (2016a) Abscisic acid promotes proteasome-mediated degradation of the transcription coactivator NPR1 in Arabidopsis thaliana. Plant $J$ 86:20-34.

Ding ZT, Zhang Z, Zhong J, Luo D, Zhou JY, Yang J, Xiao L, Shu D and Tan H (2016b) Comparative transcriptome analysis between an evolved abscisic acid-overproducing mutant Botrytis cinerea TBC-A and its ancestral strain Botrytis cinerea TBC-6. Sci Rep-Uk 6.

Dong X, Jiang Z, Peng YL and Zhang Z (2015) Revealing shared and distinct gene network organization in Arabidopsis immune responses by integrative analysis. Plant Physiol 167:1186-1203.

Frey NFD, Garcia AV, Bigeard J, Zaag R, Bueso E, Garmier M, Pateyron S, de Tauzia-Moreau ML, Brunaud V, Balzergue S, Colcombet J, Aubourg S, Martin-Magniette ML and Hirt H (2014) Functional analysis of Arabidopsis immune-related MAPKs uncovers a role for MPK3 as negative regulator of inducible defences. Genome Biol 15. R87.

Genot B, Lang J, Berriri S, Garmier M, Gilard F, Pateyron S, Haustraete K, Van Der Straeten D, Hirt H and Colcombet J (2017) Constitutively active Arabidopsis MAP Kinase 3 triggers defense responses involving salicylic acid and SUMM2 resistance protein. Plant Physiol. 174:1238-1249.

Gong T, Shu D, Yang J, Ding ZT and Tan H (2014) Sequencing and Transcriptional Analysis of the Biosynthesis Gene Cluster of Abscisic Acid-Producing Botrytis cinerea. Int J Mol Sci 15:17396-17410.

Gupta A, Hisano H, Hojo Y, Matsuura T, Ikeda Y, Mori IC and Senthil-Kumar M (2017) Global profiling of phytohormone dynamics during combined drought and pathogen stress in Arabidopsis thaliana reveals ABA and JA as major regulators. Sci Rep-Uk 7.4017.

Heidrich K, Wirthmueller L, Tasset C, Pouzet C, Deslandes L and Parker JE (2011) Arabidopsis EDS1 Connects Pathogen Effector Recognition to Cell Compartment-Specific Immune Responses. Science 334:14011404.

Hillmer RA, Tsuda K, Rallapalli G, Asai S, Truman W, Papke MD, Sakakibara H, Jones JDG, Myers CL and Katagiri $F$ (2017) The highly buffered Arabidopsis immune signaling network conceals the functions of its components. Plos Genet 13:e1006639. 
Kaurilind E, Xu E and Brosché M (2015) A genetic framework for $\mathrm{H} 2 \mathrm{O} 2$ induced cell death in Arabidopsis thaliana. Bmc Genomics 16.

Kettner J and Dorffling K (1995) Biosynthesis and Metabolism of Abscisic-Acid in Tomato Leaves Infected with Botrytis-Cinerea. Planta 196:627-634.

Kim Y, Tsuda K, Igarashi D, Hillmer RA, Sakakibara H, Myers CL and Katagiri F (2014) Mechanisms underlying robustness and tunability in a plant immune signaling network. Cell Host Microbe 15:84-94.

Kimura S, Waszczak C, Hunter K and Wrzaczek M (2017) Bound by Fate: Reactive Oxygen Species in Receptor-Like Kinase Signaling. Plant Cell.

Krishnaswamy S, Verma S, Rahman MH and Kav NNV (2011) Functional characterization of four APETALA2family genes (RAP2.6, RAP2.6L, DREB19 and DREB26) in Arabidopsis. Plant Mol Biol 75:107-127.

Li B, Meng XZ, Shan LB and He P (2016) Transcriptional Regulation of Pattern-Triggered Immunity in Plants. Cell Host \& Microbe 19:641-650.

Li L, Li M, Yu LP, Zhou ZY, Liang XX, Liu ZX, Cai GH, Gao LY, Zhang XJ, Wang YC, Chen S and Zhou JM (2014) The FLS2-Associated Kinase BIK1 Directly Phosphorylates the NADPH Oxidase RbohD to Control Plant Immunity. Cell Host \& Microbe 15:329-338.

Liang X, Ding P, Lian K, Wang J, Ma M, Li L, Li L, Li M, Zhang X, Chen S, Zhang Y and Zhou JM (2016) Arabidopsis heterotrimeric $\mathrm{G}$ proteins regulate immunity by directly coupling to the FLS2 receptor. Elife 5:e13568.

Liu L, Sonbol FM, Huot B, Gu YN, Withers J, Mwimba M, Yao J, He SY and Dong XN (2016) Salicylic acid receptors activate jasmonic acid signalling through a non-canonical pathway to promote effectortriggered immunity. Nat Commun 7. 13099.

Liu SA, Kracher B, Ziegler J, Birkenbihl RP and Somssich IE (2015) Negative regulation of ABA signaling by WRKY33 is critical for Arabidopsis immunity towards Botrytis cinerea 2100. Elife 4. e07295.

Liu XM, Nguyen XC, Kim KE, Han HJ, Yoo J, Lee K, Kim MC, Yun DJ and Chung WS (2013) Phosphorylation of the zinc finger transcriptional regulator ZAT6 by MPK6 regulates Arabidopsis seed germination under salt and osmotic stress. Biochem Bioph Res Co 430:1054-1059.

Luo HL, Laluk K, Lai ZB, Veronese P, Song FM and Mengiste T (2010) The Arabidopsis Botrytis Susceptible1 Interactor Defines a Subclass of RING E3 Ligases That Regulate Pathogen and Stress Responses. Plant Physiol 154:1766-1782.

Meng X, Xu J, He Y, Yang KY, Mordorski B, Liu Y and Zhang S (2013) Phosphorylation of an ERF transcription factor by Arabidopsis MPK3/MPK6 regulates plant defense gene induction and fungal resistance. Plant Cell 25:1126-1142.

Meng XZ and Zhang SQ (2013) MAPK Cascades in Plant Disease Resistance Signaling. Annu Rev Phytopathol 51:245-266.

Mengiste T, Chen X, Salmeron J and Dietrich R (2003) The BOTRYTIS SUSCEPTIBLE1 gene encodes an $R 2 R 3 M Y B$ transcription factor protein that is required for biotic and abiotic stress responses in Arabidopsis. Plant Cell 15:2551-2565.

Mersmann S, Bourdais G, Rietz S and Robatzek S (2010) Ethylene Signaling Regulates Accumulation of the FLS2 Receptor and Is Required for the Oxidative Burst Contributing to Plant Immunity. Plant Physiol 154:391-400.

Mignolet-Spruyt L, Xu E, Idanheimo N, Hoeberichts FA, Muhlenbock P, Brosche M, Van Breusegem F and Kangasjarvi J (2016) Spreading the news: subcellular and organellar reactive oxygen species production and signalling. $J$ Exp Bot 67:3831-3844.

Miller G, Schlauch K, Tam R, Cortes D, Torres MA, Shulaev V, Dangl JL and Mittler R (2009) The plant NADPH oxidase RBOHD mediates rapid systemic signaling in response to diverse stimuli. Sci Signal 2:ra45.

Mithoe SC, Ludwig C, Pel MJC, Cucinotta M, Casartelli A, Mbengue M, Sklenar J, Derbyshire P, Robatzek S, Pieterse CMJ, Aebersold R and Menke FLH (2016) Attenuation of pattern recognition receptor signaling is mediated by a MAP kinase kinase kinase. Embo Rep 17:441-454.

Penninckx IAMA, Thomma BPHJ, Buchala A, Metraux JP and Broekaert WF (1998) Concomitant activation of jasmonate and ethylene response pathways is required for induction of a plant defensin gene in Arabidopsis. Plant Cell 10:2103-2113. 
Perez-Salamo I, Papdi C, Rigo G, Zsigmond L, Vilela B, Lumbreras V, Nagy I, Horvath B, Domoki M, Darula Z, Medzihradszky K, Bogre L, Koncz C and Szabados L (2014) The Heat Shock Factor A4A Confers Salt Tolerance and Is Regulated by Oxidative Stress and the Mitogen-Activated Protein Kinases MPK3 and MPK6. Plant Physiol 165:319-334.

Plett JM, Cvetkovska M, Makenson P, Xing T and Regan S (2009) Arabidopsis ethylene receptors have different roles in Fumonisin B-1-induced cell death. Physiol Mol Plant P 74:18-26.

Qiu JL, Fiil BK, Petersen K, Nielsen HB, Botanga CJ, Thorgrimsen S, Palma K, Suarez-Rodriguez MC, Sandbech-Clausen S, Lichota J, Brodersen P, Grasser KD, Mattsson O, Glazebrook J, Mundy J and Petersen M (2008) Arabidopsis MAP kinase 4 regulates gene expression through transcription factor release in the nucleus. Embo J 27:2214-2221.

Rao MV, Lee H and Davis KR (2002) Ozone-induced ethylene production is dependent on salicylic acid, and both salicylic acid and ethylene act in concert to regulate ozone-induced cell death. Plant Journal 32:447-456.

Rasmussen MW, Roux M, Petersen M and Mundy J (2012) MAP kinase cascades in Arabidopsis innate immunity. Front Plant Sci 3.

Rasmussen S, Barah P, Suarez-Rodriguez MC, Bressendorff S, Friis P, Costantino P, Bones AM, Nielsen HB and Mundy J (2013) Transcriptome responses to combinations of stresses in Arabidopsis. Plant Physiol 161:1783-1794.

Rejeb IB, Pastor V and Mauch-Mani B (2014) Plant Responses to Simultaneous Biotic and Abiotic Stress: Molecular Mechanisms. Plants (Basel) 3:458-475.

Robert-Seilaniantz A, Grant M and Jones JD (2011) Hormone crosstalk in plant disease and defense: more than just jasmonate-salicylate antagonism. Annu Rev Phytopathol 49:317-343.

Serrano M, Coluccia F, Torres M, L'Haridon F and Metraux JP (2014) The cuticle and plant defense to pathogens. Front Plant Sci 5. 274.

Sham A, Moustafa K, Al-Ameri S, Al-Azzawi A, Iratni R and AbuQamar S (2015) Identification of Arabidopsis Candidate Genes in Response to Biotic and Abiotic Stresses Using Comparative Microarrays. Plos One 10. e0125666

Sharon A, Elad Y, Barakat R and Tudzynski P (2007) Phytohormones In Botrytis-Plant Interactions, in Botrytis: Biology, Pathology and Control (Elad Y, Williamson B, Tudzynski P and Delen N eds), Springer Netherlands, Dordrecht. $p^{\wedge} p p$ 163-179.

Shaul O, Elad Y and Zieslin N (1996) Suppression of Botrytis blight in cut rose flowers with gibberellic acid. Effects of exogenous application of abscisic acid and paclobutrazol. Postharvest Biol Tec 7:145-150.

Siewers V, Kokkelink L, Smedsgaard J and Tudzynski P (2006) Identification of an abscisic acid gene cluster in the grey mold Botrytis cinerea. Appl Environ Microb 72:4619-4626.

Siewers V, Smedsgaard J and Tudzynski P (2004) The p450 monooxygenase BcABA1 is essential for abscisic acid biosynthesis in Botrytis cinerea. Appl Environ Microb 70:3868-3876.

Spoel SH, Johnson JS and Dong X (2007) Regulation of tradeoffs between plant defenses against pathogens with different lifestyles. P Natl Acad Sci USA 104:18842-18847.

Straus MR, Rietz S, van Themaat EVL, Bartsch M and Parker JE (2010) Salicylic acid antagonism of EDS1driven cell death is important for immune and oxidative stress responses in Arabidopsis. Plant Journal 62:628-640.

Sun YD, Li L, Macho AP, Han ZF, Hu ZH, Zipfel C, Zhou JM and Chai JJ (2013) Structural Basis for flg22Induced Activation of the Arabidopsis FLS2-BAK1 Immune Complex. Science 342:624-628.

Survila M, Davidsson PR, Pennanen V, Kariola T, Broberg M, Sipari N, Heino P and Palva ET (2016) Peroxidase-Generated Apoplastic ROS Impair Cuticle Integrity and Contribute to DAMP-Elicited Defenses. Front Plant Sci 7.

Taki N, Sasaki-Sekimoto Y, Obayashi T, Kikuta A, Kobayashi K, Ainai T, Yagi K, Sakurai N, Suzuki H, Masuda T, Takamiya K, Shibata D, Kobayashi Y and Ohta H (2005) 12-oxo-phytodienoic acid triggers expression of a distinct set of genes and plays a role in wound-induced gene expression in Arabidopsis. Plant Physiol 139:1268-1283. 
Tsuda K, Mine A, Bethke G, Igarashi D, Botanga CJ, Tsuda Y, Glazebrook J, Sato M and Katagiri F (2013) Dual Regulation of Gene Expression Mediated by Extended MAPK Activation and Salicylic Acid Contributes to Robust Innate Immunity in Arabidopsis thaliana. Plos Genet 9. e1004015.

Tsuda K, Sato M, Stoddard T, Glazebrook J and Katagiri F (2009) Network Properties of Robust Immunity in Plants. Plos Genet 5. e1000772.

Tuominen H, Overmyer K, Keinanen M, Kollist H and Kangasjärvi J (2004) Mutual antagonism of ethylene and jasmonic acid regulates ozone-induced spreading cell death in Arabidopsis. Plant J 39:59-69.

Vaahtera L, Brosche M, Wrzaczek M and Kangasjärvi J (2014) Specificity in ROS signaling and transcript signatures. Antioxid Redox Signal 21:1422-1441.

van Loon LC, Rep M and Pieterse CMJ (2006) Significance of inducible defense-related proteins in infected plants. Annual Review of Phytopathology 44:135-162.

Wang H, Lu YQ, Liu P, Wen W, Zhang JH, Ge XC and Xia Y (2013) The ammonium/nitrate ratio is an input signal in the temperature-modulated, SNC1-mediated and EDS1-dependent autoimmunity of nudt6-2 nudt7. Plant Journal 73:262-275.

Weiss D and Ori N (2007) Mechanisms of cross talk between gibberellin and other hormones. Plant Physiol 144:1240-1246.

Venugopal SC, Jeong RD, Mandal MK, Zhu SF, Chandra-Shekara AC, Xia Y, Hersh M, Stromberg AJ, Navarre D, Kachroo A and Kachroo P (2009) Enhanced Disease Susceptibility 1 and Salicylic Acid Act Redundantly to Regulate Resistance Gene-Mediated Signaling. Plos Genet 5. e1000545.

Wiermer M, Feys BJ and Parker JE (2005) Plant immunity: the EDS1 regulatory node. Curr Opin Plant Biol 8:383-389.

Willems P, Mhamdi A, Stael S, Storme V, Kerchev P, Noctor G, Gevaert K and Van Breusegem F (2016) The ROS Wheel: Refining ROS Transcriptional Footprints. Plant Physiol 171:1720-1733.

Windram O, Madhou P, McHattie S, Hill C, Hickman R, Cooke E, Jenkins DJ, Penfold CA, Baxter L, Breeze E, Kiddle SJ, Rhodes J, Atwell S, Kliebenstein DJ, Kim YS, Stegle O, Borgwardt K, Zhang CJ, Tabrett A, Legaie R, Moore J, Finkenstadt B, Wild DL, Mead A, Rand D, Beynon J, Ott S, Buchanan-Wollaston V and Denby KJ (2012) Arabidopsis Defense against Botrytis cinerea: Chronology and Regulation Deciphered by High-Resolution Temporal Transcriptomic Analysis. Plant Cell 24:3530-3557.

Withers J and Dong XN (2016) Posttranslational Modifications of NPR1: A Single Protein Playing Multiple Roles in Plant Immunity and Physiology. Plos Pathog 12.

Wituszynska W, Slesak I, Vanderauwera S, Szechynska-Hebda M, Kornas A, Van Der Kelen K, Muhlenbock P, Karpinska B, Mackowski S, Van Breusegem F and Karpinski S (2013) LESION SIMULATING DISEASE1, ENHANCED DISEASE SUSCEPTIBILITY1, and PHYTOALEXIN DEFICIENT4 Conditionally Regulate Cellular Signaling Homeostasis, Photosynthesis, Water Use Efficiency, and Seed Yield in Arabidopsis. Plant Physiol 161:1795-1805.

Wrzaczek M, Brosche M and Kangasjärvi J (2013) ROS signaling loops - production, perception, regulation. Curr Opin Plant Biol 16:575-582.

Xu E, Vaahtera L and Brosche M (2015a) Roles of Defense Hormones in the Regulation of Ozone-Induced Changes in Gene Expression and Cell Death. Mol Plant 8:1776-1794.

Xu E, Vaahtera L, Horak H, Hincha DK, Heyer AG and Brosche M (2015b) Quantitative trait loci mapping and transcriptome analysis reveal candidate genes regulating the response to ozone in Arabidopsis thaliana. Plant Cell Environ 38:1418-1433.

Zandalinas SI, Mittler R, Balfagon D, Arbona V and Gomez-Cadenas A (2017) Plant adaptations to the combination of drought and high temperatures. Physiol Plant.

Zhu JK (2016) Abiotic Stress Signaling and Responses in Plants. Cell 167:313-324. 
A

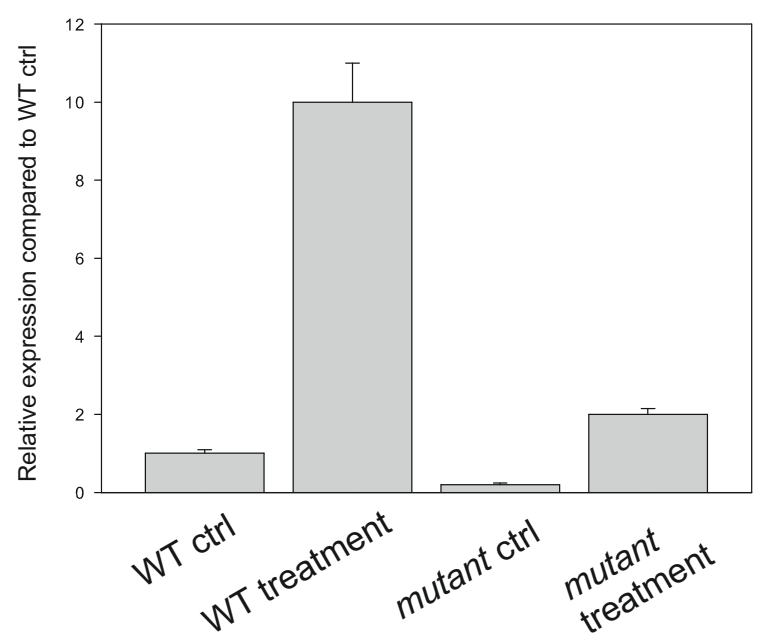

B

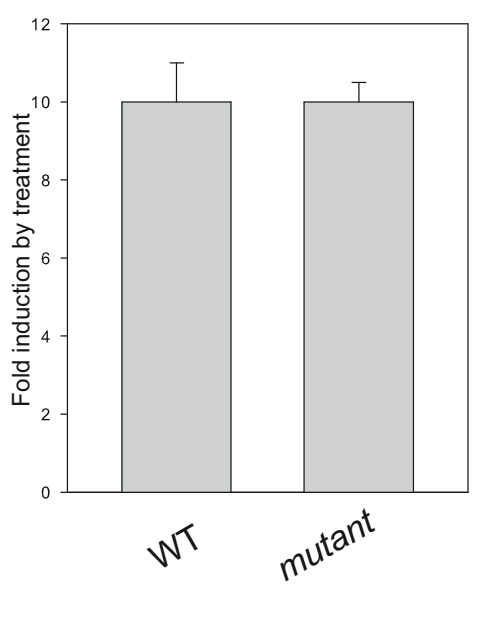



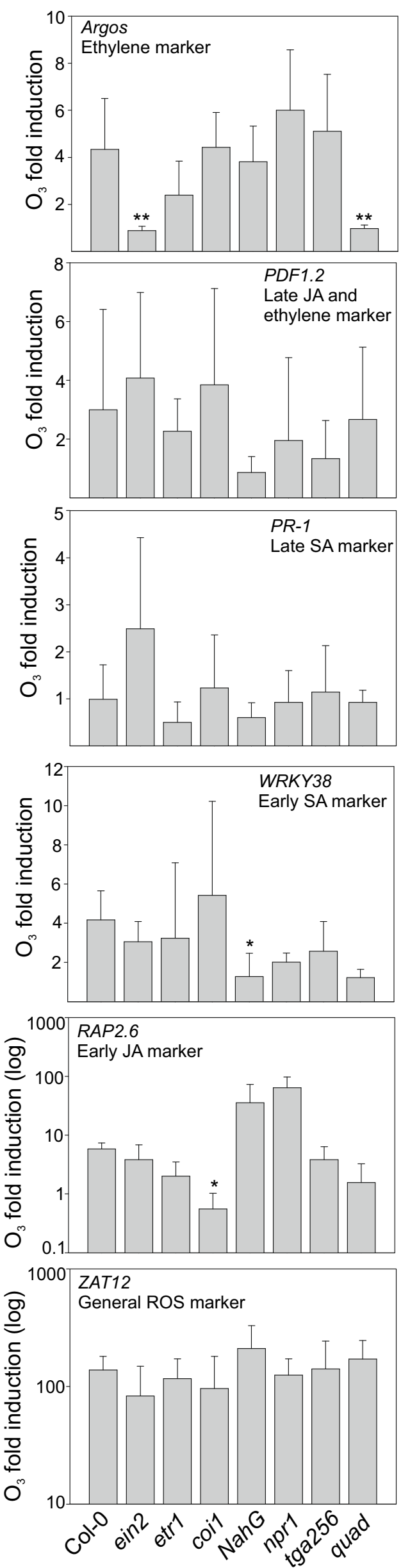

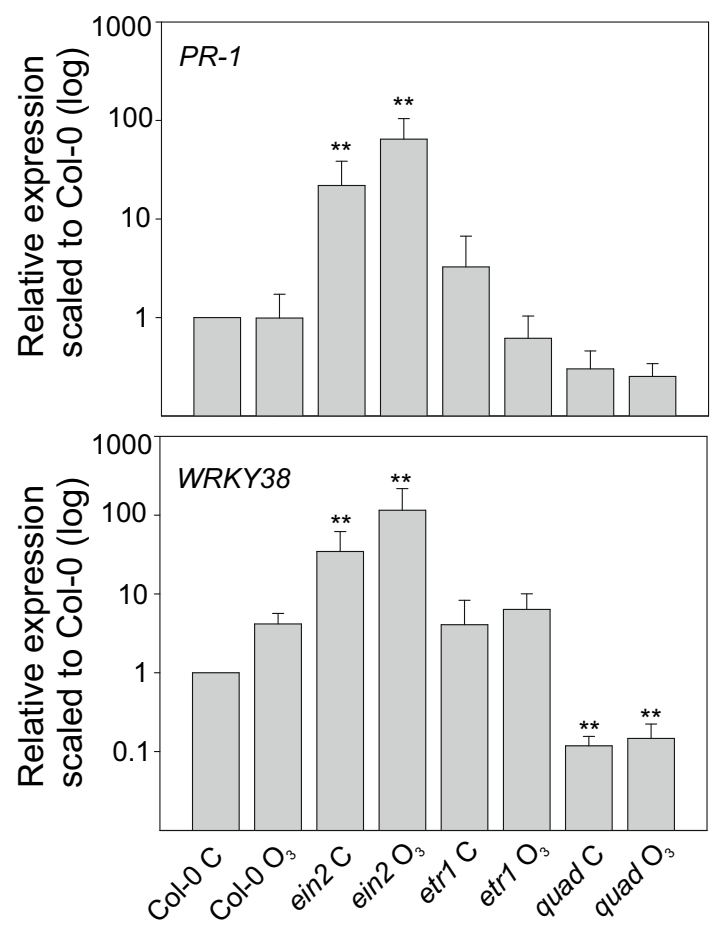